\title{
Tunable zero-order drug delivery systems created by modified triaxial electrospinning
}

\author{
Xinkuan Liu ${ }^{1, a, *}$, YaoyaoYang ${ }^{1, a}$, Deng-Guang Yu ${ }^{1, *}$, Ming-Jie Zhu ${ }^{1}$, \\ Min Zhao ${ }^{2}$, Gareth R Williams ${ }^{3}$
}

1 School of Materials Science \& Engineering, University of Shanghai for Science and Technology, Shanghai 200093, China.

2 School of Pharmacy, Queen's University Belfast, Belfast BT9 7BL, UK.

3 UCL School of Pharmacy, University College London, 29-39 Brunswick Square, London WC1N 1AX, UK.

${ }^{1}$ These authors contributed equally to this work.

\section{*Corresponding authors:}

Prof. Deng-Guang Yu and Prof. Xinkuan Liu

\section{Address:}

School of Materials Science \& Engineering, University of Shanghai for Science and Technology, 516 Jungong Road, Yangpu District, Shanghai 200093, P.R. China

Tel: +86-21-55270632

Fax: +86-21-55270632

Email: ydg017@usst.edu.cn (DGY); xinkuanliu@usst.edu.cn (XL) 


\section{ABSTRACT}

The ability to accurately control a material's structure, and in turn its functional performance, is of crucial importance for potential applications. In this study, a new modified triaxial electrospinning process was successfully developed to allow us to precisely tune drug release from nanoscale formulations. In this process, we used two un-electrospinnable liquids as the outer and middle working fluids, with only the core solution being individually electrospinnable into fibers. The outer liquid comprised a mixture of solvents, while the middle fluid was a dilute solution of cellulose acetate (CA). The core fluid was an electrospinnable co-dissolving solution of ferulic acid (FA) and gliadin. By processing these in triaxial electrospinning, we were able to create FA-gliadin fibers coated with a thin but even and continuous coating of CA. The thickness of the CA coating could be precisely varied by adjusting the flow rate of the middle working fluid. The resultant nanofibers have linear and cylindrical morphologies with clear core-shell structures. X-ray diffraction and IR spectroscopy data verified that the fibers comprised amorphous solid dispersions, with intermolecular interactions existing between FA and gliadin. The CA coating eliminated the initial burst release seen with uncoated FA-gliadin fibers, and also led to close to zero-order release profiles which could be incrementally adjusted by varying the thickness of the coating. New process-nanostructure-performance relationships are therefore revealed, and the advanced triaxial electrospinning approach reported in this work has great potential for developing new kinds of functional nanomaterials.

Keywords: Modified triaxial electrospinning; Polymer nanocoating; Tunable zero-order drug release; Fluid flow rate; Structural nanohybrid 


\section{Introduction}

Adaptable techniques able to create advanced materials with tunable properties are highly sought after in a wide variety of fields [1-3]. Electrospinning is one such technique. It employs electrical energy to draw and solidify polymer-containing solutions or melts, yielding products in the form of one-dimensional nanoscale fibers. Over the past two decades, this one-step "top-down" process has quickly developed. Initial studies focused on the simplest monoaxial experiment, employing a mixed solution of a polymer and functional component to generate monolithic fibers [4-9]. More recently, researchers have developed two-fluid side-by-side and coaxial electrospinning processes (resulting in Janus or core/shell fibers) [10-16], three-fluid triaxial electrospinning, and other more complicated experiments [17-20].

Control of the materials produced by electrospinning can be obtained through the processing parameters. There is abundant scope to vary these through the working fluids (viscosity and surface tension), the operational parameters (applied voltage, needle-to-collector distance and fluid flow rate), and the environmental conditions (humidity, temperature, etc) [21]. Electrospinning is able to create complex nanostructures via the macroscale structure of the spinneret: this is propagated into the architecture of the fibers produced, and thus use of a single needle to dispense the liquid being processed results in monolithic fibers, while using a coaxial spinneret (with one needle nested inside a second) yields core-shell materials. Processing multiple fluids simultaneously has another advantage because it allows liquids which cannot be electrospun individually to be processed into fibers, if they are spun 
alongside a spinnable fluid. Thus, multiple-fluid electrospinning methods are powerful in generating composites with a range of complex nanostructures, such as core-shell materials, Janus structures, core-shell systems with a Janus core, or Janus fibers with one monolithic and one core-shell side [22-27].

Among the various nanoscale architectures which can be envisaged, the core-shell arrangement is probably the most fundamental, and has been investigated for potential applications in numerous scientific fields [28-31]. Methods to fabricate core-shell structures have been very widely explored: a Web of Science with "core-shell" as the topic conducted on 29 April 2018 resulted in 32,988 hits in the preceding 5 years, equating to 18 publications per day. Coaxial electrospinning and coaxial electrospraying offer the ability to generate core-shell particles or core-shell nanofibers in a single step, and thus can be advantageous over other multi-step nanofabrication approaches in terms of simplicity and ease of implementation $[32,33]$. The fact that only one of the fluids need be electrospinnable in coaxial experiments leads to a broad range of possible products [34-37].

Building on the concept of coaxial electrospinning, triaxial electrospinning has in recent years begun to attract attention [38-41]. In the same way that in coaxial electrospinning only one fluid need be individually electrospinnable, unspinnable fluids can be processed by triaxial electrospinning so long as a spinnable fluid is also being dispensed. In the standard triaxial electrospinning method all three fluids are spinnable, but a number of modified processes including one or more unspinnable liquids can be envisaged depending on which of the fluids (outer, middle, inner; 
combinations thereof) are spinnable. Two unspinnable fluids and one spinnable fluid can be combined in such modified triaxial procedures, which is very beneficial because there exists a limitless number of unspinnable fluids, while only around one hundred polymers can be directly electrospun into nanofibers. As a result, there are a large number of possibilities for generating structural nanomaterials $[34,40]$. To date, the literature suggests that if two unspinnable solutions are being processed they should be separated: i.e., unspinnable outer and inner liquids can be electrospun if combined with a middle fluid which can be spun into fibers alone, but the process will not be viable if the outer and middle (or middle and inner) solutions are both unspinnable. In this work, we vitiate this hypothesis and report a new modified triaxial electrospinning process in which the outer fluid is an unspinnable solvent mixture, the middle fluid an unspinnable dilute polymer solution, and only the inner solution is electrospinnable.

In the development of new drug delivery systems, much effort has been expended to achieve so-called controlled release (for instance, pulsatile, sustained, bi-phasic or targeted release) of active pharmaceutical ingredients (APIs) [42-45]. In particular, linear drug release (or zero-order release) is much sought after. The constant rate of drug release offered by such systems allows for a constant, therapeutically active, concentration of API to be maintained over a prolonged period of time, with no over- or under-dosing. Unfortunately, electrospun drug-loaded polymer nanocomposites tend to exhibit an uncontrollable initial burst of release [46-47]. This arises for three reasons: (1) the presence of large numbers of drug 
molecules at or near the surface of the fibers; (2) the large surface area and high porosity of the fiber mats; and (3) the fact that drug molecules in the center of the fibers have to diffuse further to reach the bulk solution during the dissolution process than those at the surface. Core-shell nanostructures have been shown to have the ability to eliminate this effect, in general by confining the drug to the core of the fiber such that it has to diffuse through an insoluble shell $[48,49]$. However, in traditional coaxial electrospinning the shell thickness can be difficult to control.

In this work, a modified triaxial electrospinning process was developed to prepare drug-loaded fibers coated with a thin polymer shell layer. The latter comprised cellulose acetate (CA), and was applied to a ferulic acid (FA)-loaded protein (gliadin) core. FA is an antioxidant that can be exploited to neutralize free radicals (including nitric oxide, superoxide and hydroxyl radicals). It is reported to have the potential to reduce the risk of many cancers (e.g. liver, colon, stomach, breast, prostate, lung and tongue), and also to decrease blood glucose levels (meaning it has promise for the treatment of diabetes) [50,51]. FA is poorly soluble, and its sustained release would be useful to improve its absorption and bioavailability [40, 52]. The materials reported here provide zero-order drug release profiles, and the release duration and rate can be tuned through the thickness of the CA coating, which can itself be precisely manipulated through the flow rate of the middle fluid in electrospinning. 


\section{Experimental}

\subsection{Materials}

Ferulic acid (FA; 4-hydroxy-3-methoxycinnamic acid) was purchased from Shanghai Yu-Bo Biological Technology Co., Ltd. (Shanghai, China). Gliadin (extracted from wheat) was obtained from the Miao-Sheng Biotechnology Co., Ltd. (Shanghai, China). Cellulose acetate $\left(\mathrm{CA} ; M_{\mathrm{w}}=100,000 \mathrm{Da}\right)$ was supplied by Acros (NJ, USA). Colorants (methylene blue and basic fuchsin) and analytical grade solvents (1,1,1,3,3,3-hexafluoro-2-propanol (HFIP), trifluoroacetic acid (TFA), acetone and acetic acid) were purchased from the Shanghai Zi-Yi Chem. Co., Ltd. (Shanghai, China). Water was doubly distilled before use.

\subsection{Electrospinning}

The triaxial electrospinning system comprised three syringe pumps (KDS100, Cole-Parmer, Vernon Hills, IL, USA), a high-voltage generator (ZGF60kV/2mA, Wuhan Huatian Co., Ltd., Wuhan, China), a tri-layer concentric spinneret, and a flat piece of cardboard wrapped with aluminum foil used as the collector. The electrospinning processes were filmed using a Canon camera (PowerShot SX50HS, Tokyo, Japan).

\subsection{Morphology and structure}

The nanofibers' morphology was studied with the aid of a Quanta FEG450 scanning electron microscope (FE-SEM; FEI Corporation, Hillsboro, OR, USA). Samples were platinum sputter-coated under a nitrogen atmosphere for $100 \mathrm{~s}$ prior to visualization. Images were recorded at an excitation voltage of $20 \mathrm{kV}$ with a magnification of 5,000. Fiber diameters were calculated by using the ImageJ software (National Institutes of Health, Bethesda, MD, USA) to measure the fibers at 100 different points. 
Internal structure was explored with a transmission electron microscope (TEM; JEM 2100F, JEOL, Tokyo, Japan) at an excitation voltage of $300 \mathrm{kV}$. TEM samples were prepared by fixing a lacey carbon-coated copper grid on the collector and depositing fibers directly on it.

\subsection{Component compatibility and physical form}

X-ray diffraction (XRD) patterns were collected on a D/Max-BR diffractometer (Rigaku, Tokyo, Japan) over the $2 \theta$ range 5 to $60^{\circ}$. The instrument was supplied with $\mathrm{Cu} \mathrm{K \alpha}$ radiation at $40 \mathrm{mV}$ and $30 \mathrm{~mA}$. The compatibility of the fiber components (FA, CA and gliadin) was evaluated through attenuated total reflectance-Fourier transform infrared spectroscopy (ATR-FTIR; Nicolet-Nexus 670 spectrometer, Nicolet Instrument Corporation, Madison, WI, USA). Spectra were obtained from 500-4000 $\mathrm{cm}^{-1}$ with a resolution of $2 \mathrm{~cm}^{-1}$ and 8 scans collected per sample.

\subsection{Drug sustained release performances}

FA has a maximum UV absorbance at $\lambda_{\max }=321 \mathrm{~nm}$ and a shoulder at $278 \mathrm{~nm}$. A calibration curve was thus constructed at $321 \mathrm{~nm}$ with the aid of a Lambda $750 \mathrm{~S}$ spectrophotometer (Perkin Elmer, Waltham, MA, USA). This took the form $C=$ $17.74 A+0.14\left(\mathrm{R}^{2}=0.9996\right)$, where $C$ is the concentration of FA $(\mu \mathrm{g} / \mathrm{mL})$ and $A$ is the absorbance (linear range: $1-20 \mu \mathrm{g} / \mathrm{mL}$ ).

Drug release was quantified following the Chinese Pharmacopoeia Method II (a paddle method) on RCZ-8A dissolution apparatus (Tianjin University Radio Factory, Tianjin, China) at $50 \mathrm{rpm}$ and $37^{\circ} \mathrm{C} .30 \mathrm{mg}$ of each sample was placed into $600 \mathrm{~mL}$ of phosphate buffered saline (PBS, $\mathrm{pH}=7.0,0.1 \mathrm{M}$ ). At predetermined time points, $5 \mathrm{~mL}$ aliquots were withdrawn, and $5 \mathrm{~mL}$ of fresh preheated PBS was added to the to maintain a constant volume. The absorbance of the aliquots at $\lambda_{\max }=321 \mathrm{~nm}$ was used to determine the amount of FA released at each time point (with suitable dilution 
performed where required, to ensure the absorbance lay within the calibration range) Dissolution tests for each sample were performed six times, and the results are reported as mean \pm S.D.

\section{Results and discussion}

\subsection{Modified triaxial electrospinning}

In the traditional monoaxial electrospinning process, the working fluid must be electrospinnable to generate polymer nanofibers. Similarly, in coaxial electrospinning, it was once thought that the shell fluid must be spinnable $[53,54]$. However, Yu et al. showed that the latter was not the case, allowing the development of a modified coaxial process in which unspinnable shell liquids can be employed to create both core-shell and high quality monolithic nanocomposites (using a pure solvent as the shell working fluid) [23]. Building on this, Yang et al. developed modified triaxial electrospinning, using an electrospinnable middle liquid to support both outer and inner unspinnable fluids and resulting in core-shell polymer-phospholipid/drug nanohybrids [34]. Yang and co-workers also hypothesized that it would not be viable to implement a modified triaxial electrospinning process where both the outer and middle fluids could not be electrospun alone.

In this study, we disproved this hypothesis and developed a new modified triaxial process in which the outer fluid was a mixture of solvents (which cannot be electrospun), the middle fluid an unspinnable dilute polymer solution, and only the core can be processed into fibers (see Fig. 1). The apparatus used comprises four main components (Fig. 1a): the power supply; a three-layer concentric spinneret (Fig. 1b and c); a collector plate; and, three pumps for expelling the working fluids independently. The use of the dilute polymer middle solution results in the core being 
coated in a very thin polymer shell. The presence of solvent at the outer layer helps to stabilize the working processes from the following three aspects: 1) preventing the clinging of CA solution on the nozzle of spinneret; 2) resisting the negative influences from the environment; and 3) providing an easy commencing of the triaxial process.

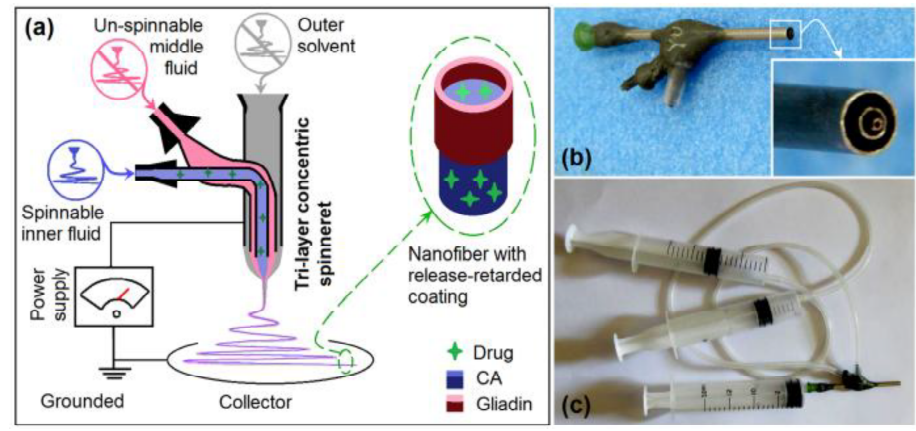

Fig. 1. The modified triaxial electrospinning apparatus: (a) a schematic diagram of the components and their connectivity; (b) the triaxial concentric spinneret; (c) the connection of the spinneret with the working fluids.

A digital photograph of the electrospinning apparatus is shown in Fig. 2a. Color markers were added to enable the process to be easily visualized. The middle solution contained basic fuchsin $\left(5 \times 10^{-6} \mathrm{~g} / \mathrm{mL}\right)$ and the inner fluid methylene blue $\left(5 \times 10^{-6}\right.$ $\mathrm{g} / \mathrm{mL}$ ), giving them pink and blue colors respectively. Four different fiber formulations were prepared (detailed in Table 1). For the preparation of F1, the middle fluid flow rate was set to zero, and a mixture of acetic acid and acetone used for the outer liquid - this results in a modified coaxial experiment. The core fluid contained gliadin and FA. An image of a typical coaxial working process is given in Fig. 2b, while Fig. 2c depicts the compound Taylor cone (with the inner fluid encapsulated by the outer solvent), followed by continuous bending and whipping loops. 

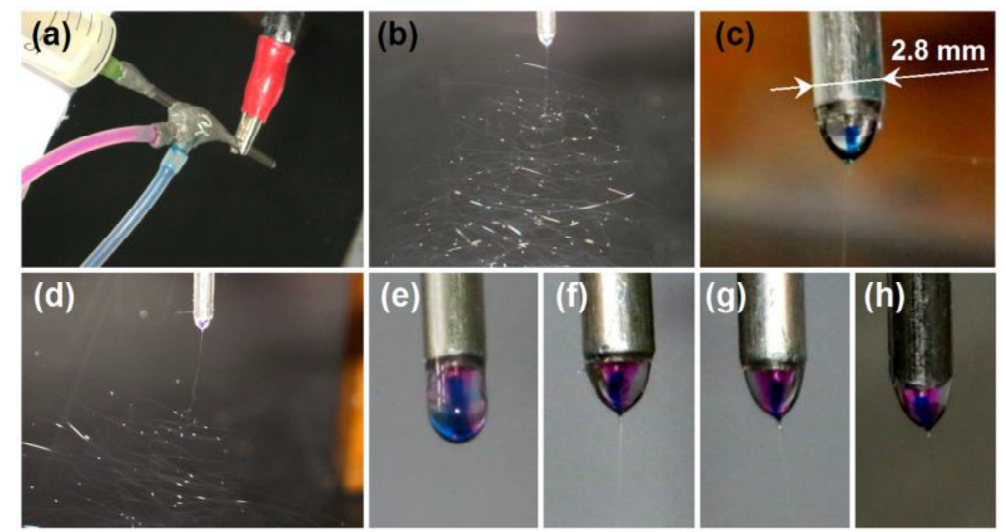

Fig 2. Images of the electrospinning processes: (a) the connection of the spinneret with the power supply; (b) a typical modified coaxial electrospinning process as used to prepare F1, with (c) the compound Taylor cone; (d) a typical modified triaxial electrospinning experiment; (e) the three-layer droplet obtained when no electrical charge was applied; and the compound Taylor cones seen at $15 \mathrm{kV}$ with (f) F3; (g) F2; (h) and F4.

Images obtained during the preparation of F3 are presented in Fig. 2d, 2e and 2f. The modified triaxial process shows the usual three stages observed during the conversion of the working fluids into solid products (Fig. 2d). When no voltage is applied, a three-layer droplet is seen (Fig. 2e). Upon application of a voltage of $15 \mathrm{kV}$, a compound Taylor cone (Fig. 2f) is obtained. A straight fluid jet is emitted from the tip of the cone (Fig. 2d), after which the unstable whipping and bending region is observed. This consists of gradually enlarging loops, due to electrical repulsion between the different parts of the jet. Similar compound Taylor cones are seen for F2 and F4 (see Fig. 2f, 2h). For a smooth modified triaxial electrospinning, the spinnable core fluid guided the formation of nanofibers with two unspinnable fluids. Thus, its electrospinnability is very important and the concentration of filament-forming carrier is a key parameter, just as the modified coaxial electrospinning [55]. A practicable strategy is to slightly elevate the concentration of filament-forming carrier within its 
electrospinnable window. In literature, the gliadin concentration was $15 \%(\mathrm{w} / \mathrm{v})$ for a modified coaxial process [24]. Here, a concentration of $16 \%(\mathrm{w} / \mathrm{v})$ was intentionally exploited for a robust modified triaxial process and nanofibers without beads-on-a-string morphology.

Table 1 The experimental parameters used for preparing nanofibers in this work. The applied voltage was $15 \mathrm{kV}$, and the spinneret to collector distance $20 \mathrm{~cm}$.

\begin{tabular}{|c|c|c|c|c|c|c|c|}
\hline \multirow{2}{*}{ No. } & \multirow{2}{*}{$\begin{array}{l}\text { Working } \\
\text { process }\end{array}$} & \multirow{2}{*}{$\begin{array}{l}\text { Middle } \\
\text { fluid }\end{array}$} & \multicolumn{3}{|c|}{ Fluid flow rate $(\mathrm{mL} / \mathrm{h})$} & \multirow{2}{*}{ Structure } & \multirow{2}{*}{$\begin{array}{l}\text { Sheath thickness }^{\mathrm{c}}(\mathrm{nm}) \\
\quad(\text { Mean } \pm \text { S.D. })\end{array}$} \\
\hline & & & Outer $^{a}$ & Middle & Inner $^{b}$ & & \\
\hline $\mathrm{F} 1$ & $\begin{array}{c}\text { Modified } \\
\text { coaxial }\end{array}$ & - & 0.3 & -- & 2 & Monolithic & 0 \\
\hline $\mathrm{F} 2$ & \multirow{3}{*}{$\begin{array}{l}\text { Modified } \\
\text { triaxial }\end{array}$} & \multirow{3}{*}{$\begin{array}{l}6 \% \mathrm{w} / \mathrm{v} \\
\mathrm{CA}\end{array}$} & 0.3 & 0.1 & 2 & Core-shell & $5.2 \pm 2.6$ \\
\hline F3 & & & 0.3 & 0.2 & 2 & Core-shell & $14.7 \pm 1.6$ \\
\hline $\mathrm{F} 4$ & & & 0.3 & 0.5 & 2 & Core-shell & $30.2 \pm 10.1$ \\
\hline
\end{tabular}

${ }^{a}$ The outer working fluid comprised a mixture of acetone and acetate acid $(2: 1 \mathrm{v} / \mathrm{v})$, and the middle CA solutions were prepared using the same solvent mixture.

${ }^{\mathrm{b}}$ The inner working fluid contained $4 \%(\mathrm{w} / \mathrm{v})$ FA and $16 \%(\mathrm{w} / \mathrm{v})$ gliadin in a solvent mixture of HFIP and TFA (8:2 v/v).

${ }^{\mathrm{c}}$ The sheath thickness was estimated from the TEM images by over 10 places in Fig. 4.

\subsection{Fiber morphology and structure}

All the fibers produced have linear cylindrical morphologies, with smooth surfaces (as exhibited in Fig. 3). No beads-on-a-string or spindles-on-a-string phenomena are visible. As the flow rate of the middle solution (CA in acetone: acetate acid $(2: 1 \mathrm{v} / \mathrm{v}))$ increased from $0(\mathrm{~F} 1)$, to $0.1(\mathrm{~F} 2), 0.2(\mathrm{~F} 3)$ and to $0.5 \mathrm{~mL} / \mathrm{h}(\mathrm{F} 4)$, the fibers produced showed a decrease in their diameters, from $0.86 \pm 0.12 \mu \mathrm{m}(\mathrm{F} 1)$, to $0.74 \pm 0.09 \mu \mathrm{m}(\mathrm{F} 2), 0.68 \pm 0.10 \mu \mathrm{m}(\mathrm{F} 3)$, and $0.63 \pm 0.09 \mu \mathrm{m}(\mathrm{F} 4)$

An increase in the middle fluid flow rate is expected to exert two opposing influences on the fiber diameters. The first is to enlarge the fibers, because more 
solute is dispensed through the spinneret per unit time. The second is to allow the working fluids to be drawn under the electric field for a longer period of time, since the CA solution is too dilute to be electrospinnable. It is clear that the second factor dominates over the first.

CA is derived from a natural biopolymer, and it is known that such polymers (e.g. zein and ethylcellulose) have a tendency to form gels and generate semi-solid substances around the exit of the spinneret during electrospinning. This phenomenon appears even at low polymer concentrations [56]. In this study, the outer solvent prevents any clinging and build-up of semi-solid CA on the spinneret, allowing a stable and continuous working process to be implemented. The presence of a pure solvent as the outer fluid also helps the inner and middle fluids to be drawn evenly under the electrical field [57]. Thus, just as modified coaxial electrospinning aids in the preparation of high quality monolithic nanocomposites (e.g. F1), the outer solvent in modified triaxial electrospinning helps to generate high quality core-shell hybrids (F2 to F4).
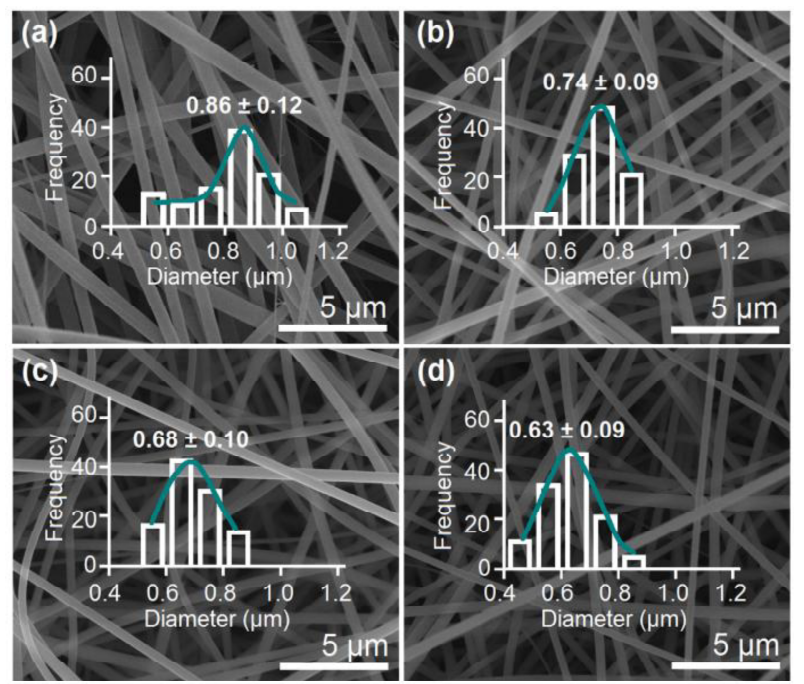

Fig 3. SEM images of the nanofibers: (a) F1; (b) F2; (c) F3; (d) F4. 
The internal structures of the fibers F1 to F4 were explored using TEM (Fig. 4). F1 comprise drug-protein monolithic nanocomposites, which is reflected in the even gray color observed in Fig. 4a. No particles or solid phase separation can be seen. This suggests that the FA molecules were evenly dispersed throughout the gliadin matrix.

In contrast, nanofibers F2, F3 and F4 have obvious core-shell structures, as shown in Fig. 4b, 4c and 4d, respectively. The CA coatings on F2 to F4 are estimated to have thicknesses of $5.2 \pm 2.6,14.7 \pm 1.6,30.2 \pm 10.1 \mathrm{~nm}$. In all cases, although the shell thickness varies somewhat within each formulation, the core FA-gliadin composite is completely enveloped by the CA coating.

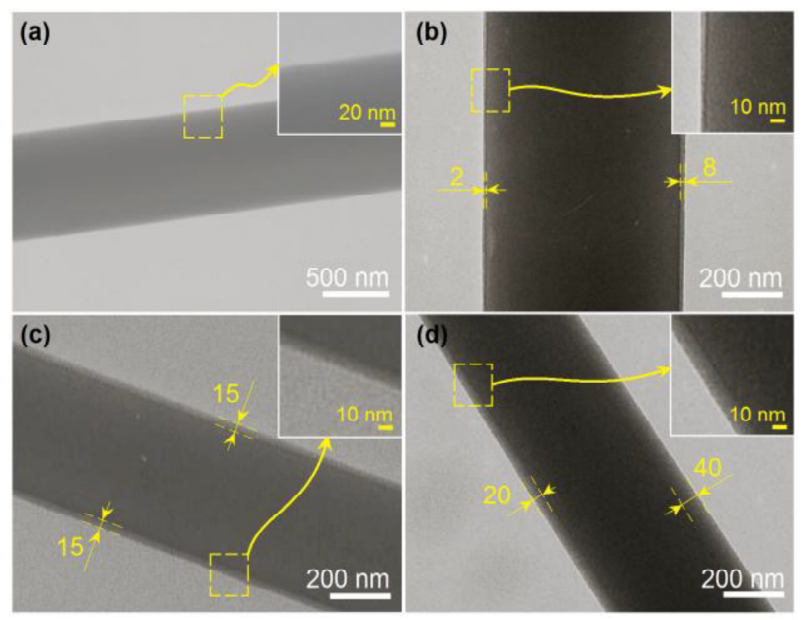

Fig 4. TEM images of the fibers: (a) F1; (b) F2; (c) F3; (d) F4.

If the core FA-gliadin and the shell CA coating are assumed to have an equal density, a theoretical value of the shell thicknesses can be calculated using the following equation:

$$
\frac{Q_{\mathrm{s}}}{Q_{f}}=\frac{\left(R_{f}-R_{c}\right)^{2} L_{f} \pi}{R_{f}^{2} L_{f} \pi}=\frac{F_{s} \times C_{s}}{\left(F_{c} \times C_{c}+F_{s} \times C_{s}\right)}
$$


where $Q, R, L, F$, and $C$ respectively represent the mass of material dispensed, the fiber radius and length, the fluid flow rate and the solute concentration, and the subscript $s, f$, and $c$ represent the shell, the whole fiber and the core. $R_{f}$ can easily be calculated from the known fiber diameter (determined by SEM). On the basis of the flow rates and concentrations, $R_{c}$ is calculated for F2, F3 and F4 to be 367.2, 335.0, and $303.8 \mathrm{~nm}$. The CA coating thicknesses are found to be $2.8(\mathrm{~F} 2), 5.0(\mathrm{~F} 3)$, and 11.2 nm (F3), respectively. These values are all two or three times smaller than the measured values from TEM. The difference likely arises because of differences in the core and shell densities; the latter is likely to be lower than the former, as is indicated by the lower contrast level in the TEM images.

\subsection{Physical form and component compatibility}

Drug-loaded nanofibers from the monoaxial electrospinning of a mixed drug-polymer solution have received broad interest for enhancing the dissolution of poorly water-soluble drugs, since they typically comprise amorphous solid dispersions [58]. The formation of an amorphous material can also be helpful for controlling release [59]. XRD patterns for the raw materials and fibers are depicted in Fig. 5. FA is a crystalline material, as demonstrated by a series of sharp Bragg reflections in its XRD pattern. In contrast, both CA and gliadin are amorphous, and display only broad halos in XRD. All the fibers also exhibited only halos, showing that they too are amorphous. This arises because of the extremely fast drying which occurs during electrospinning; this causes the disordered arrangement of molecules in solution to be propagated into the solid state. 


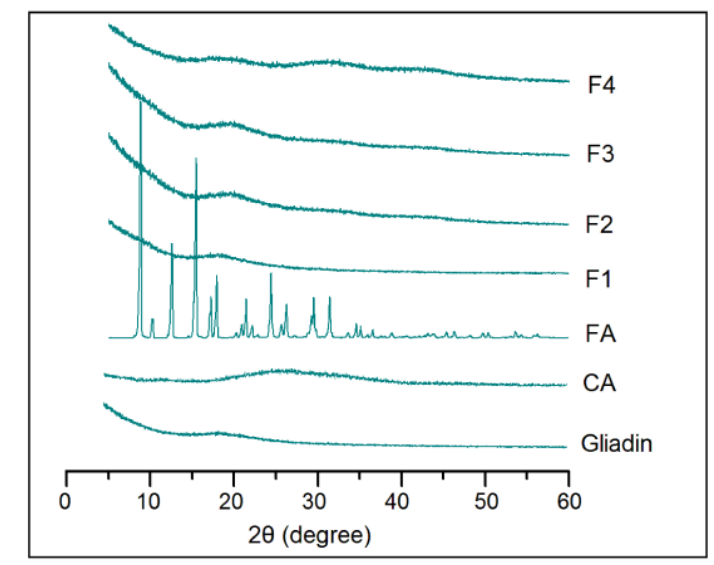

Fig 5. XRD patterns of the raw materials and the fibers.

The molecular structures of the raw materials and IR spectra are included in Fig. 6. FA has a number of characteristic bands at $1789,1663,1619$, and $1514 \mathrm{~cm}^{-1}$, which correspond to vibrations of its $-\mathrm{C}=\mathrm{O}$ groups and benzene rings. These peaks cannot be seen in the spectrum of the FA-gliadin F1 fibers, suggesting that secondary interactions are formed between the two components. These might include hydrogen bonds (e.g. between gliadin $-\mathrm{OH} /-\mathrm{NH}$ groups and $-\mathrm{C}=\mathrm{O}$ on FA) and hydrophobic interactions (between the alkyl groups of gliadin and benzene rings), and suggest there is a high degree of compatibility between the components. This should enhance the physical stability of the drug-gliadin composites. Looking at the IR spectra of the core-shell fibers F2, F3 and F4, it is clear that they contain some additional peaks, for instance at $1724 \mathrm{~cm}^{-1}, 1236 \mathrm{~cm}^{-1}$, and $1051 \mathrm{~cm}^{-1}$. These correspond to $\mathrm{CA}$, and suggest that the shell CA and core FA-gliadin are present as two-component hybrids. As the thicknesses of the CA coating increased from F2 to F4, the intensity of the CA peaks increased correspondingly. This observation can be closely related to use of attenuated reflectance IR in these measurements: the penetration depth of the IR probe 
in this technique is around $200 \mathrm{~nm}$. Thus, the increase in the shell thickness corresponds to a decrease in the amount of the core illuminated.

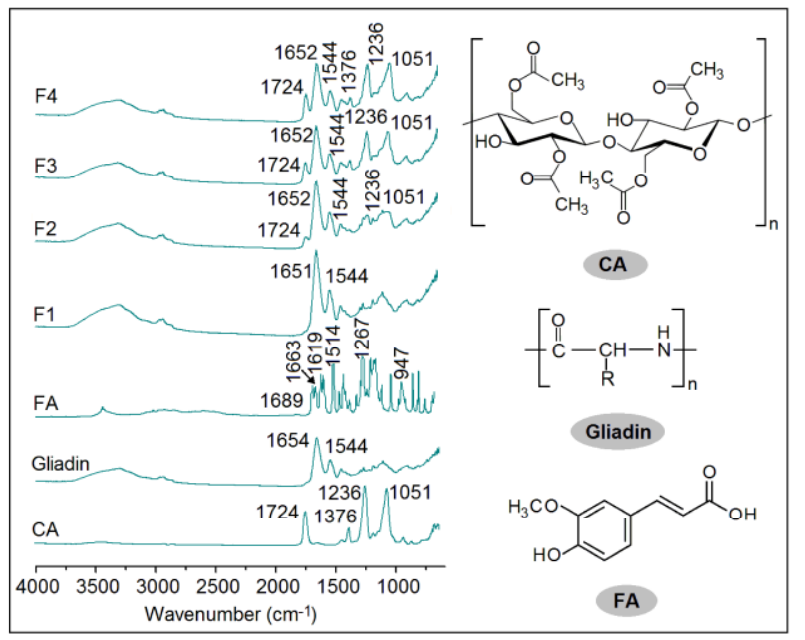

Fig. 6. The molecular formulae of the raw materials, and IR spectra.

\subsection{In vitro drug release}

The in vitro FA release profiles are shown in Fig. 7a. It is clear that the time taken for release gradually increased with the thicknesses of the CA coating. An enlargement of the first $120 \mathrm{~min}$ is presented in Fig. 7b. After the first fifteen minutes, $\mathrm{F} 1, \mathrm{~F} 2, \mathrm{~F} 3$ and F4 released $20.3 \pm 5.1 \%, 1.5 \pm 1.2 \%, 0 \%$, and $0 \%$ of the incorporated FA. After the first hour, the release percentages are $37.2 \pm 4.5 \%, 15.4 \pm 4.6 \%, 7.6 \pm$ $4.1 \%$, and $3.1 \pm 2.2 \%$, respectively. The monolithic FA-gliadin F1 material clearly undergoes a significant initial burst of release. In contrast, the core-shell systems F2 to F4 show no initial burst release, regardless of the thicknesses of the CA coating. Thus, the CA coating on the FA-gliadin nanocomposites can both extend the release time periods and eliminate of the initial burst release.

The zero-order equation was first fitted to the release data. The results are exhibited in Fig. 7c. For F1 to F4, the linear equations obtained are: $Q_{1}=27.06+6.97 t$ 
$\left(R_{1}=0.8858\right), \quad Q_{2}=9.21+5.56 t \quad\left(\mathrm{R}_{2}=0.9887\right), \quad Q_{3}=9.31+3.09 t \quad\left(\mathrm{R}_{3}=0.9804\right), \quad$ and $Q_{4}=4.04+2.59 t\left(\mathrm{R}_{4}=0.9899\right)$, respectively. Both the correlation coefficients and visual inspection of the plots (Fig. 7c) show that the core-shell hybrids F2 to F4 provide close to zero order release, while the fit is poor for F1.

To further explore the release mechanisms, the data were analyzed using the Korsmeyer-Peppas expression [60]:

$Q=\frac{M_{\mathrm{t}}}{M_{\infty}}=\mathrm{kt}^{n}$

where $M_{\mathrm{t}}$ is the amount of drug released at time $t, M_{\infty}$ is the total amount of drug released, $k$ is the rate constant, and $n$ is an exponent which provides some information on the drug release mechanism. The regressed equations for fibers F1 to F4 (Fig. 7d) are $Q_{1}=40.2 t^{0.36}\left(\mathrm{R}_{1}=0.0 .9828\right), Q_{2}=15.6 t^{0.62}\left(\mathrm{R}_{2}=0.9956\right), Q_{3}=8.5 t^{0.71} \quad\left(\mathrm{R}_{3}=0.9951\right)$, $Q_{4}=2.9 t^{0.98}\left(\mathrm{R}_{4}=0.9903\right)$, respectively. For $\mathrm{F} 1$, the value of $\mathrm{n}$ is 0.36 , smaller than the critical value of 0.45 [60]. This suggests that FA was released through a typical Fickian diffusion mechanism. All the core-shell hybrids F2 to F4 have $n>0.45$ (suggesting a combination of diffusion and erosion controls release), and for F4 n > 0.89 (suggesting an erosion-dominated mechanism). However, both the shell CA and gliadin core are insoluble in water, and so erosion is not likely to be a major contributor to release. The Peppas equation assumes that the drug is homogeneously distributed throughout the polymer matrix, which is not the case for the core-shell formulations. For the latter, it is expected that the rate of release is governed by FA diffusing through the gliadin matrix and CA shell. This will involve two different rate constants, which might be why non-Fickian mechanisms are indicated in Peppas 
analysis.

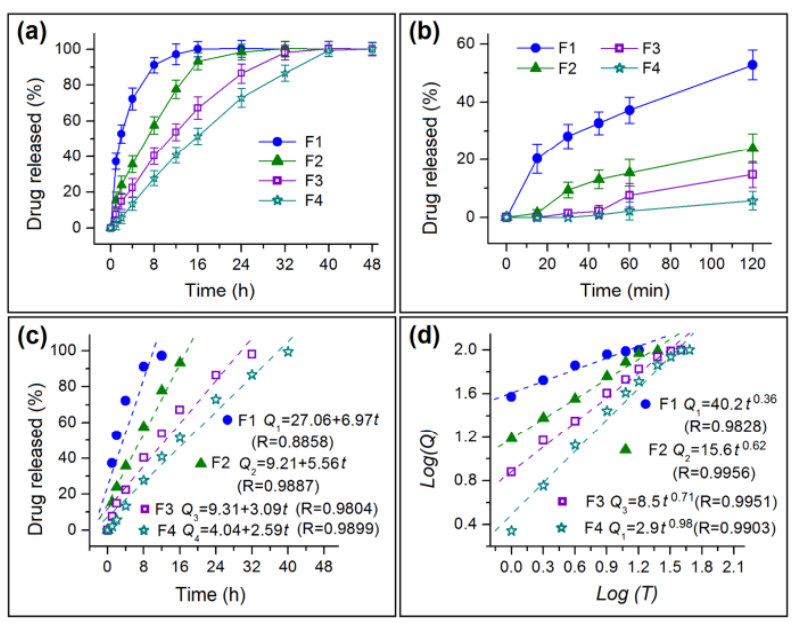

Fig. 7. In vitro dissolution results. Raw release data are given for for (a) the entire $48 \mathrm{~h}$ experiment and (b) the first $2 \mathrm{~h}$, together with fits of the (c) zero-order and (d) Peppas equations to the release data. Data are given as mean \pm S.D. from six independent experiments.

\subsection{Process-structure-performance relationships}

As noted above, electrospinning is able to create complex nanostructures in a single "top-down" step [61, 62]. Being able to obtain precise control over these materials is crucial for designing and developing new functional nanomaterials. Here, we are able to use the processing parameters to precisely tune the properties of the fibers generated. There are very clear relationships between the thickness of the CA coating and the time taken to reach different extents of release. The time taken reach $30 \%, 50 \%$ and $90 \%$ release is plotted as a function of the coating thickness in Fig. 8a. Regressed linear equations of $t_{30 \%}=1.38+0.25 T_{\mathrm{S}} \quad(\mathrm{R}=0.9880), \quad t_{50 \%}=3.34+0.43 T_{\mathrm{S}}$ $(\mathrm{R}=0.9731)$, and $t_{90 \%}=10.15+0.86 T_{\mathrm{S}}(\mathrm{R}=0.9727)$ can be fitted to the data, where $t_{X X \%}$ (in min) is the time taken to reach 30,50 , or $90 \%$ release and $T_{\mathrm{S}}$ is the thickness of the CA coating (in $\mathrm{nm}$ ). 
The thickness of the CA coating is itself closely correlated with the flow rate of the middle fluid $(F)$, as can be seen in Fig. $8 \mathrm{~b}$. A linear equation of the form $T_{\mathrm{S}}=0.36+60.71 \mathrm{~F}(\mathrm{R}=0.9915)$ can be determined. This simple relationship means that it will be possible to produce a wide range of materials with a particular coating thickness, and therefore a desired release time, in a stable and robust manner. The drug release times (Fig. 8b) also show approximately linear relationships with the flow rate of the middle fluid as follows: $t_{30 \%}=1.50+15.34 F \quad(\mathrm{R}=0.9880)$, $t_{50 \%}=3.55+25.71 F(\mathrm{R}=0.9551)$, and $t_{90 \%}=10.69+51.07 F(\mathrm{R}=0.9426)$.
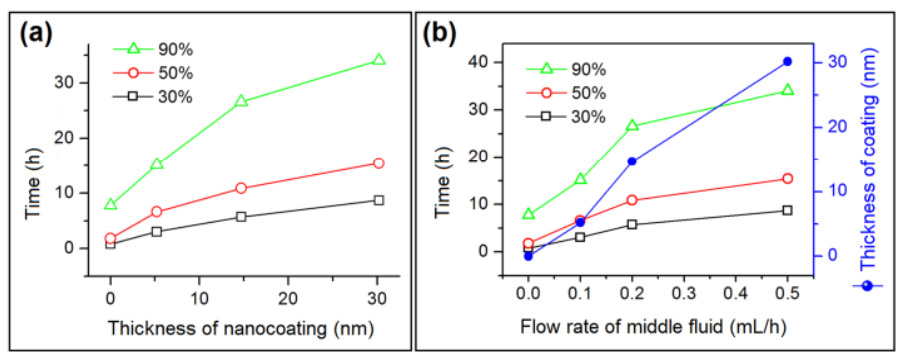

Fig. 8. Process-structure-performance relationships between (a) the CA coating thickness and the release times; (b) the middle fluid flow rate, coating thickness, and release times.

In many drug-polymer composites, including those reported in this work, the drug is molecularly distributed throughout the polymer matrix, as shown for F1 in Fig. 9. This distribution means that the drug release profile is mainly determined by the physical and chemical properties of the carrier. Even when using an insoluble carrier to achieve sustained release profiles, because of the presence of a substantial quantity of drug at or near the surface of the composite, an initial burst release and eventual tailoring-off are inevitable. Very significant efforts have been made to overcome these issues, including through the fabrication of a range of electrospun nanofibers $[63,64]$. 


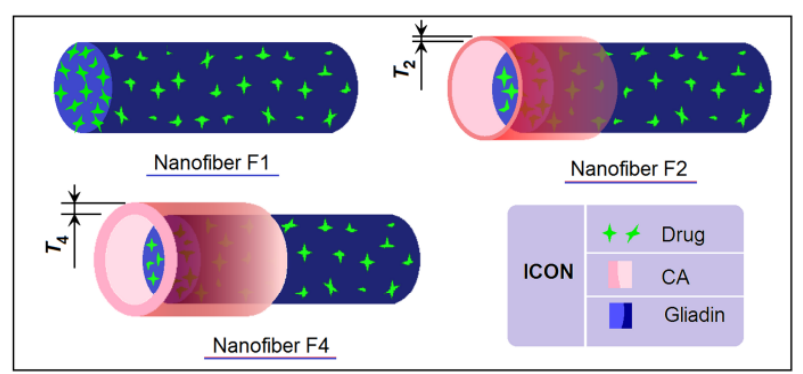

Fig. 9. A schematic diagram showing the structures and the drug distributions of the nanofibers prepared in this work.

In the core-shell nanohybrids prepared by modified triaxial electrospinning (F2, F3, F4), a blank CA coating was applied to yield a product with a heterogeneous distribution of drug (Fig. 9). The CA coating thickness can be very easily controlled, and alternative coatings (for instance, biodegradable, water-soluble or water-swelling polymers) could also be applied. Since the coating thickness directly influences the drug release profile in a predictable manner, by varying the flow rate of the central fluid used for spinning we can directly dictate the release profile through simple control of a single processing parameter.

Both electrospinning and electrospraying take advantage of the interactions between electrostatic energy and the working fluids. Thus, the strategies reported here can also be adopted to develop new triaxial electrospraying processes for creating new nanostructures, for tailoring components and compositions, and for a combined application of different kinds of functional ingredients $[65,66]$. As for the further developments of the medicated nanofibers, both production on a large scale, conversion to dosage forms and also the related stability should be the contents deserved intensive investigations [67-69].

\section{Conclusions}


In this study, we successfully implemented a new triaxial electrospinning process. In this, we used two unspinnable liquids as the outer and middle working fluids and a spinnable core, and were able to produce high-quality fibers. The outer fluid comprised a mixture of acetone and acetic acid, while the middle fluid was a dilute cellulose acetate (CA) solution. Neither can be electrospun info fibers alone, but the use of an electrospinnable ferulic acid (FA)-gliadin solution as the core fluid permitted successful nanofiber fabrication. As a result, we were able to generate high-quality core-shell nanofibers with a thin CA coating and an FA-loaded core. These all comprised amorphous solid dispersions, with intermolecular interactions believed to exist between the drug and gliadin. While monolithic FA-gliadin fibers exhibited a profound initial burst of release, the presence of a CA coating could completely eliminate this. The core-shell materials gave close to zero-order drug release over time periods which could be tuned simply by adjusting the thicknesses of the CA shell. The latter could in turn be incrementally varied by changing the flow rate of the middle working fluid in triaxial electrospinning. There is thus a very clear process-structure-performance relationship, which offers myriad opportunities for designing and fabricating novel structural nanomaterials with improved functional performance.

\section{Acknowledgements}

This work was financially supported by the National Natural Science Foundation of China (grant number 51373101).

\section{References}

[1] B.P. Isaacofft, K.A. Brown, Progress in top-down control of bottom-up assembly, 
Nano Lett. 17 (2017) 6508-6510.

[2] J.J. Kaufman, G.M. Tao, S. Shabahang, E.H. Banaei, D.S.S. Deng, X.D. Liang, S.G. Johnson, Y. Fink, A.F. Abouraddy, Structured spheres generated by an in-fibre fluid instability, Nature 489 (2012) 463-467.

[3] L.J. Lauhon, M.S. Gudiksen, C.L. Wang, C.M. Lieber, Epitaxial core-shell and core-multishell nanowire heterostructures, Nature 420 (2002) 57-61.

[4] S. Jiang, Y. Chen, G. Duan, C. Mei, A. Greiner, S. Agarwal, Electrospun nanofiber reinforced composites: A review, Polym. Chem. 2018, http://dx.doi.org/10.1039/C8PY00378E.

[5] T. Zhu, K. Yu, M.A. Bhutto, X. Guo, W. Shen, J. Wang, W. Chen, H. El-Hamshary, S.S. Al-Deyab, X. Mo, Synthesis of RGD-peptide modified poly(ester-urethane) urea electrospun nanofibers as a potential application for vascular tissue engineering, Chem. Eng. J. 315 (2017) 177-190.

[6] F. Xu, H. Sheardown, T. Hoare, Reactive electrospinning of degradable poly(oligoethylene glycol methacrylate)-based nanofibrous hydrogel networks, Chem. Commun. 52 (2016) 1451-1454.

[7] P. Mehta, R. Haj-Ahmad, M. Rasekh, M.S. Arshad, A. Smith, S.M. van der Merwe, X. Li, M.W. Chang, Z. Ahmad, Pharmaceutical and biomaterial engineering via electrohydrodynamic atomization technologies, Drug Discov. Today 22 (2017) 157-165.

[8] D.D. Wang, J.N. Wang, Electrospinning Polyvinyl alcohol/silica-based nanofiber as highly efficient adsorbent for simultaneous and sequential removal of Bisphenol A and Cu(II) from water, Chem. Eng. J. 314 (2017) 714-726.

[9] S. Lee, M.K. Joshi, A.P. Tiwari, B. Maharjan, K.S. Kim, Y.-H. Yun, C.H. Park, C.S. Kim, Lactic acid assisted fabrication of bioactive three-dimensional PLLA/ $\beta$-TCP fibrous scaffold for biomedical application, Chem. Eng. J. 347 (2018) 771-781.

[10]K.T. Shalumon, C. Sheu, C.-H. Chen, S.-H. Chen, G. Jose, C.-Y. Kuo, J.-P. Chen, Multi-functional electrospun antibacterial core-shell nanofibrous membranes for prolonged prevention of post-surgical tendon adhesion and inflammation, Acta Biomater. 72 (2018) 121-136.

[11]M.W. Lee, S. An, Y.I. Kim, S.S. Yoon, A.L. Yarin, Self-healing three-dimensional bulk materials based on core-shell nanofibers, Chem. Eng. J. 334 (2018) 
1093-1100.

[12]Z.C. Yao, C.C. Zhang, Z. Ahmad, J. Huang, J.S. Li, M.W. Chang, Designer fibers from $2 \mathrm{D}$ to $3 \mathrm{D}$-Simultaneous and controlled engineering of morphology, shape and size, Chem. Eng. J. 334 (2018) 89-98.

[13]K. Wang, X.K. Liu, X.H. Chen, D.G. Yu, Y.Y. Yang, P. Liu, Electrospun hydrophilic janus nanocomposites for the rapid onset of therapeutic action of helicid, ACS Appl. Mater. Interfaces 10 (2018) 2859-2867.

[14]Y.W. Liu, Q.L. Ma, M. Yang, X.T. Dong, Y. Yang, J.X. Wang, W.S. Yu, G.X. Liu, Flexible hollow nanofibers: Novel one-pot electrospinning construction, structure and tunable luminescence-electricity-magnetism trifunctionality, Chem. Eng. J. 284 (2016) 831-840.

[15]Y. Gao, D. Zhao, M.W. Chang, Z. Ahmad, J.S. Li, Optimising the shell thickness-to-radius ratio for the fabrication of oil-encapsulated polymeric microspheres, Chem. Eng. J. 284 (2016) 963-971.

[16]S. An, Y. Il Kim, H.S. Jo, M.W. Kim, M.W. Lee, A.L. Yarin, S.S. Yoon, Silver-decorated and palladium-coated copper-electroplated fibers derived from electrospun polymer nanofibers, Chem. Eng. J. 327 (2017) 336-342.

[17]D. Han, S. Sherman, S. Filocamo, A.J. Steckl, Long-term antimicrobial effect of nisin released from electrospun triaxial fiber membranes, Acta Biomater. 53 (2017) 242-249.

[18]S. Jiang, G. Duan, E. Zussman, A. Greiner, S. Agarwal, Highly flexible and tough concentric triaxial polystyrene fibers, ACS Appl. Mater. Interfaces 6 (2014) 5918-5923.

[19]S. Labbaf, H. Ghanbar, E. Stride, M. Edirisinghe, Preparation of multilayered polymeric structures using a novel four-needle coaxial electrohydrodynamic device, Macromol. Rapid Commun. 35 (2014) 618-623.

[20]Z. Ahmad, H.B. Zhang, U. Farook, M. Edirisinghe, E. Stride, P. Colombo, Generation of multilayered structures for biomedical applications using a novel tri-needle coaxial device and electrohydrodynamic flow, J. R. Soc. Interface 5 (2008) 1255-1261.

[21]S. Agarwal, A. Greiner, J.H. Wendorff, Functional materials by electrospinning of polymers, Prog. Polym. Sci. 38 (2013) 963-991. 
[22]J.D. Starr, M.A.K. Budi, J.S. Andrew, Processing-property relationships in electrospun janus-type biphasic ceramic nanofibers, J. Am. Ceram. Soc. 98 (2015) 12-19.

[23]Q. Wang, D.G. Yu, L.L. Zhang, X.K. Liu, Y.C. Deng, M. Zhao, Electrospun hypromellose-based hydrophilic composites for rapid dissolution of poorly water-soluble drug, Carbohyd. Polym. 174 (2017) 617-625.

[24]D.G. Yu, J.J. Li, G.R. Williams, M. Zhao, Electrospun amorphous solid dispersions of poorly water-soluble drugs: A review, J. Control. Release 2018 https://doi.org/10.1016/j.jconrel.2018.08.016

[25]D.G. Yu, J.J. Li, M. Zhang, G.R. Williams, High-quality Janus nanofibers prepared using three-fluid electrospinning, Chem. Commun. 53 (2017) 4542-4545.

[26]X.M. Guo, X.T. Dong, J.X. Wang, W.S. Yu, G.X. Liu, Fabrication of novel $\mathrm{La}_{2} \mathrm{O}_{2} \mathrm{CN}_{2}$ one-dimensional nanostructures via facile electrospinning combined with cyanamidation technique, Chem. Eng. J. 250 (2014) 148-156.

[27]W.W. Liu, C.Y. Ni, D.B. Chase, J.F. Rabolt, Preparation of multilayer biodegradable nanofibers by triaxial electrospinning, ACS Macro Lett. 2 (2013) 466-468

[28]J. Gao, W.X. Kong, L.Y. Zhou, Y. He, L. Ma, Y. Wang, L.Y. Yin, Y.J. Jiang, Monodisperse core-shell magnetic organosilica nanoflowers with radial wrinkle for lipase immobilization, Chem. Eng. J. 309 (2017) 70-79.

[29]M. Naeimirad, A. Zadhoush, R. Kotek, R.E. Neisiany, S.N. Khorasani, S. Ramakrishna, Recent advances in core/shell bicomponent fibers and nanofibers: A review, J. Appl. Polym. Sci. 135 (2018).

[30] Y.Z. Chang, L.Z. He, Z.B. Li, L.L. Zeng, Z.H. Song, P.H. Li, L. Chan, Y.Y. You, X.F. Yu, P.K. Chu, T.F. Chen, Designing core-shell gold and selenium nanocomposites for cancer radiochemotherapy, ACS Nano 11 (2017) 4848-4858.

[31]C.T. Chen, X. Chen, H. Zhang, Q. Zhang, L. Wang, C.X. Li, B.B. Dai, J.Z. Yang, J. Liu, D.P. Sun, Electrically-responsive core-shell hybrid microfibers for controlled drug release and cell culture, Acta Biomater. 55 (2017) 434-442.

[32]I.G. Loscertales, A. Barrero, I. Guerrero, R. Cortijo, M. Marquez, A.M. Ganan-Calvo, Micro/nano encapsulation via electrified coaxial liquid jets, Science 295 (2002) 1695-1698. 
[33]Y. Dzenis, Material science. Spinning continuous fibers for nanotechnology, Science 304 (2004) 1917-1919.

[34]C. Yang, D.G. Yu, D. Pan, X.K. Liu, X. Wang, S.W.A. Bligh, G.R. Williams, Electrospun $\mathrm{pH}$-sensitive core shell polymer nanocomposites fabricated using a tri-axial process, Acta Biomater. 35 (2016) 77-86.

[35]Z.P. Liu, Y.Y. Zhang, D.G. Yu, D. Wu, H.L. Li, Fabrication of sustained-release zein nanoparticles via modified coaxial electrospraying, Chem. Eng. J. 334 (2018) 807-816.

[36]X.Y. Li, Z.B. Zheng, D.G. Yu, X.K. Liu, Y.L. Qu, H.L. Li, Electrosprayed sperical ethylcellulose nanoparticles for an improved sustained-release profile of anticancer drug, Cellulose 24 (2017) 5551-5564.

[37]K. Wang, H.F. Wen, D.G. Yu, Y.Y. Yang, D.F. Zhang, Electrosprayed hydrophilic nanocomposites coated with shellac for colon-specific delayed drug delivery, Mater. Design 143 (2018) 248-255.

[38]D.G. Yu, X.Y. Li, X. Wang, J.H. Yang, S.W.A. Bligh, G.R. Williams, Nanofibers fabricated using triaxial electrospinning as zero order drug delivery systems, ACS Appl. Mater. Interface 7 (2015) 18891-18897.

[39]D. Han, A.J. Steckl, Triaxial electrospun nanofiber membranes for controlled dual release of functional molecules, ACS Appl. Mater. Interface 5 (2013) 8241-8245.

[40]G.Z. Yang, J.J. Li, D.G. Yu, M.F. He, J.H. Yang, G.R. Williams, Nanosized sustained-release drug depots fabricated using modified tri-axial electrospinning, Acta Biomater. 53 (2017) 233-241.

[41]J.D. Starr, J.S. Andrew, A route to synthesize multifunctional tri-phasic nanofibers, J. Mater. Chem. C 1 (2013) 2529-2533.

[42]S. Campbell, D. Maitland, T. Hoare, Enhanced pulsatile drug release from injectable magnetic hydrogels with embedded thermosensitive microgels, Acs Macro Lett. 4 (2015) 312-316.

[43]D. Maitland, S.B. Campbell, J. Chen, T. Hoare, Controlling the resolution and duration of pulsatile release from injectable magnetic 'plum-pudding' nanocomposite hydrogels, RSC Adv. 6 (2016) 15770-15781.

[44]U. Angkawinitwong, S. Awwad, P.T. Khaw, S. Brocchini, G.R. Williams, Electrospun formulations of bevacizumab for sustained release in the eye, Acta 
Biomater. 64 (2017) 126-136.

[45]S. Mitragotri, P.A. Burke, R. Langer, Overcoming the challenges in administering biopharmaceuticals: formulation and delivery strategies, Nat. Rev. Drug Discov. 13 (2014) 655-672.

[46]S. Qi, D. Craig, Recent developments in micro-and nanofabrication techniques for the preparation of amorphous pharmaceutical dosage forms, Adv. Drug Deliv. Rev. 100 (2016) 67-84.

[47]Z. Mao, J. Li, W. Huang, H. Jiang, B.L. Zimba, L. Chen, J. Wan, Q. Wu, Preparation of poly(lactic acid)/graphene oxide nanofiber membranes with different structures by electrospinning for drug delivery, RSC Adv. 8 (2018) 16619-16625.

[48]N. Kamaly, B. Yameen, J. Wu, O.C. Farokhzad, Degradable controlled-release polymers and polymeric nanoparticles: mechanisms of controlling drug release, Chem. Rev. 116 (2016) 2602-2663.

[49]M. Irani, G. Mir Mohamad Sadeghi, I. Haririan, Electrospun biocompatible poly ( $\varepsilon$-caprolactonediol)-based polyurethane core/shell nanofibrous scaffold for controlled release of temozolomide, Int. J. Polym. Mater. 67 (2018) 361-366.

[50]P. Hassanzadeh, F. Atyabi, R. Dinarvand, Nanoencapsulation: A promising strategy for biomedical applications of ferulic acid, Biomed. Rev. 28 (2017) 22-30.

[51]E. Mhillaj, S. Catino, F.M. Miceli, R. Santangelo, L. Trabace, V. Cuomo, C. Mancuso, Ferulic acid improves cognitive skills through the activation of the heme oxygenase system in the rat, Mol. Neurobiol. 55 (2018) 905-916.

[52]B. Poornima, P.S. Korrapati, Fabrication of chitosan-polycaprolactone composite nanofibrous scaffold for simultaneous delivery of ferulic acid and resveratrol, Carbohyd. Polym. 157 (2017) 1741-1749.

[53]A.K. Moghe, B.S. Gupta, Co-axial electrospinning for nanofiber structures: Preparation and applications, Polym. Rev. 48 (2008) 353-377.

[54]H.L. Qu, S.Y. Wei, Z.H. Guo, Coaxial electrospun nanostructures and their applications, J. Mater. Chem. A 1 (2013) 11513-11528.

[55]J.J. Li, Y.Y. Yang, D.G. Yu, Q. Du, X.L. Yang, Fast dissolving drug delivery membrane based on the ultra-thin shell of electrospun core-shell nanofibers, Eur. 
J. Pharm. Sci. 122 (2018) 195-204.

[56]Z.P. Liu, L.L. Zhang, Y.Y. Yang, D. Wu, G. Jiang, D.G. Yu, Preparing composite nanoparticles for immediate drug release by modifying electrohydrodynamic interfaces during electrospraying, Powder Technol. 327 (2018) 179-187.

[57]Q. Wang, D.-G. Yu, S.-Y. Zhou, C. Li, M. Zhao, Electrospun amorphous medicated nanocomposites fabricated using a Teflon-based concentric spinneret, e-Polymers 18 (2018) 3-11.

[58]E. Borbás, Z.K. Nagy, B. Nagy, A. Balogh, B. Farkas, O. Tsinman, K. Tsinman, B. Sinkó, The effect of formulation additives on in vitro dissolution-absorption profile and in vivo bioavailability of telmisartan from brand and generic formulations, Eur. J. Pharm. Sci. 114 (2018) 310-317.

[59]E. Szabó, B. Démuth, B. Nagy, K. Molnár, A. Farkas, B. Szabó, A. Balogh, E. Hirsch, B. Nagy, G. Marosi, Z.K. Nagy, Scaled-up preparation of drug-loaded electrospun polymer fibres and investigation of their continuous processing to tablet form, Express Polym. Lett. 12 (2018) 436-451.

[60]N.A. Peppas, Analysis of Fickian and non-Fickian drug release from polymers, Pharm. Acta Helv. 60 (1985) 110-111.

[61]M.W. Lee, S. An, C. Lee, M. Liou, A.L. Yarin, S.S. Yoon, Self-healing transparent core-shell nanofiber coatings for anti-corrosive protection, J. Mater. Chem. A 2 (2014) 7045-7053.

[62]M. He, H. Jiang, R. Wang, Y. Xie, C. Zhao, Fabrication of metronidazole loaded poly ( $\varepsilon$-caprolactone)/zein core/shell nanofiber membranes via coaxial electrospinning for guided tissue regeneration, J. Colloid Interf. Sci. 490 (2017) 270-278.

[63] S.J. Song, Y.C. Shin, S.E. Kim, I.K. Kwon, J.H. Lee, S.H. Hyon, D.W. Han, B. Kim, Aligned laminin core-polydioxanone/collagen shell fiber matrices effective for neuritogenesis, Sci. Rep. 8 (2018) 5570.

[64]F. Nasehi, M. Karshenas, S. Nadri, G. Barati, A. Salim, Core-shell fibrous scaffold as a vehicle for sustained release of retinal pigmented epithelium-derived factor (PEDF) for photoreceptor differentiation of conjunctiva mesenchymal stem cells, J. Biomed. Mater. Res. A 105 (2017) 3514-3519.

[65]C. Zhang, Z.C. Yao, Q. Ding, J.J. Choi, Z. Ahmad, M.W. Chang, J.S. Li, Tri-needle coaxial electrospray engineering of magnetic polymer yolk-shell 
particles possessing dual-imaging modality, multiagent compartments, and trigger release potential, ACS Appl. Mater. Interf. 9 (2017) 21485-21495.

[66]E. Sayed, C. Karavasili, K. Ruparelia, R. Haj-Ahmad, G. Charalambopoulou, T. Steriotis, D. Giasafaki, P. Cox, N. Singh, L.-P.N. Giassafaki, A. Mpenekou, C.K. Markopoulou, I.S. Vizirianakis, M.-W. Chang, D.G. Fatouros, Z. Ahmad, Electrosprayed mesoporous particles for improved aqueous solubility of a poorly water soluble anticancer agent: in vitro and ex vivo evaluation, J. Control. Release 278 (2018) 142-155.

[67]A. Balogh, A. Domokos, B. Farkas, A. Farkas, Z. Rapi, D. Kiss, Z. Nyiri, Z. Eke, G. Szarka, R. Örkényi, B. Mátravölgyi, F. Faigl, G. Marosi, Z.K. Nagy, Continuous end-to-end production of solid drug dosage forms: Coupling flow synthesis and formulation by electrospinning, Chem. Eng. J. 350 (2018) 290-299.

[68]R. Haj-Ahmad, M. Rasekh, K. Nazari, E.V. Onaiwu, B. Yousef, S. Morgan, D. Evans, M.W. Chang, J. Hall, C. Samwell, Z. Ahmad, Stable increased formulation atomization using a multi-tip nozzle device. Drug Deliv. Transl. Re. 2018, https://doi.org/10.1007/s13346-018-0518-4

[69]B. Démuth, A. Farkas, H. Pataki, A. Balogh, P.L. Sóti, T. Vigh, B. Farkas, J. Mensch, G. Verreck, I. Van Assche, G. Marosi, Z.K. Nagy, Detail stability investigation of amorphous solid dispersions prepared by single-needle and high speed electrospinning, Int. J. Pharm. 498 (2016) 234-244. 